\title{
Connectedness underlies the underestimation of the horizontal vertical illusion in $\mathbf{L}$-shaped configurations
}

\author{
Yongchun $\mathrm{Cai}^{1} \cdot \mathrm{Ci} \mathrm{Wang}^{1} \cdot \mathrm{Chao} \mathrm{Song}^{1} \cdot \mathrm{Zhi} \mathrm{Li}^{1}$
}

Published online: 17 March 2017

(C) The Psychonomic Society, Inc. 2017

\begin{abstract}
L-shaped configuration is a commonly used stimulus configuration in studying horizontal vertical illusion. Here, we report that the horizontal vertical illusion is substantially underestimated when the L-shaped configuration is used for evaluating the illusion. Experiment 1 found that, in a length perception task, the perceived length of a vertical bar was about $10 \%$ longer than that of a horizontal bar with the same physical size. Similar amount of HVI was found in a length comparison task, in which the length of a horizontal bar was compared to that of a vertical bar and the two bars were presented separately in space or in time. In contrast, when the length comparison task was conducted with the two bars being arranged in a connected L-shape, the illusion was halved in strength. Experiment 2 and 3 studied what might be the cause of this L-shape induced HVI-underestimation. Two factors were investigated: the connectedness of the two lines, and the $45^{\circ}$ absolute orientation or the $45^{\circ}$ inner angle information embedded in the upright isosceles L-shape. The results showed that the HVI strength was not much affected when the $45^{\circ}$ absolute orientation and the $45^{\circ}$ angle information was made useless for the length comparison task. In contrast, the illusion was significantly reduced in strength whenever the two lines were separated as compared to when they were connected. These results suggested that the connectedness of the two lines must underlie the underestimation of the horizontal vertical illusion in the L-shaped configurations.
\end{abstract}

Zhi Li

zli1@zju.edu.cn

1 Department of Psychology and Behavioral Sciences, Zhejiang University (Xixi Campus), 148 Tian Mu Shan Road,

Hangzhou, China 310028
Keywords Horizontal-vertical illusion · Length perception · Orientation perception - Within-object comparison . Between-object comparison

\section{Introduction}

A vertical line appears slightly longer than a horizontal line that has the same physical size. This phenomenon is known as the horizontal vertical illusion (HVI). Since first discovered by Fick (1851), the HVI has been investigated extensively. Two prevalent accounts proposed for the HVI were the framing account and the size-constancy-scaling hypothesis. The framing account (Avery and Day 1969; Houck et al 1972; Künnapas 1955, 1957) attributes the illusion to the fact that our visual field is an oval, with the horizontal axis being longer than the vertical axis. The monocular visual field is about $160^{\circ}$ versus $135^{\circ}$ (Spector 1990), and the binocular visual field is about $200^{\circ}$ versus $130^{\circ}$ (see Prinzmetal and Gettleman 1993). If perceived size is correlated to the relative proportion occupied by the line in the visual field, the vertical line would be estimated relatively longer. In contrast, the sizeconstancy scaling hypothesis (Girgus and Coren 1975; Gregory 1963) emphasizes the fact that a vertical line is typically perceived as receding from the observer, whereas a horizontal line is often perceived to lie in a frontal-parallel plane. The mechanism underlying size-constancy may scale the perceived size of horizontal lines and vertical lines differently. Later studies showed that both pictorial depth and framing have independent effects on the HVI (e.g. Williams and Enns 1996).

It has been shown that stimulus configuration is an important factor that strongly influences the horizontal vertical illusion (Charras and Lupiáñez 2010; Cormack and Cormack 1974; Wolfe et al. 2005). For example, Finger and Spelt 
(1947; see also Künnapas 1955) demonstrated that if the two lines were arranged in an L-shape, the resulted illusion (about $5 \%$ ) was weaker than that when the two lines were arranged in an inverted T-shape (about 10\%). It was thus proposed that the inverted-T illusion consists of two biases - the vertical bias and the bisection bias. Other types of stimuli configurations, including side-T, cross (Cormack and Cormack 1974; Künnapas 1955) or dissected-T (Landwehr 2015) have also been investigated.

Whereas stimulus configuration clearly affects the strength of the horizontal vertical illusion, the illusion can be demonstrated alone by length perception of tilted lines (e.g. Armstrong and Marks 1997; Pollock and Chapanis 1952; Teghtsoonian 1972; Verrillo and Irvin 1979). That is, the perceived length of a line is a function of the orientation of that line, with the vertical line being perceived relatively longer than the equal-sized horizontal line. What's interesting is that the strength of the HVI demonstrated by the length perception studies (>10\%; e.g. Pollock and Chapanis 1952; Teghtsoonian 1972; Verrillo and Irvin 1979) often appeared larger than that of the HVI found in the length comparison studies with Lshaped configurations (about 5\%; e.g. Avery and Day 1969).

The horizontal vertical illusion essentially reflects the difference between perceived horizontal sizes and perceived vertical sizes, although multiple sources, including framing, sizeconstancy scaling, stimulus configuration, etc., may contribute to it. The fact that HVI obtained in the length perception task is often larger than that seen in the length comparison task with L-shaped configuration suggests that the HVI is underestimated in length comparison task with L-shaped configuration. If it is the case, what might be the cause? Here, we propose two possibilities. First, the $45^{\circ}$ angle (or the $45^{\circ}$ absolute orientation) information embedded in an upright Lshape with two equal legs may help the observer in the length comparison task. Second, the connectedness of the two lines forming the L-shape may contribute to the HVIunderestimation.

When the two sides of an upright L-shape matches in size, the implicit line connecting the two ends of the L-shape becomes $45^{\circ}$ tilted from horizontal (i.e. the $45^{\circ}$ absolute orientation information), and the L-shape also forms an implicit isosceles right-triangle, containing two $45^{\circ}$ inner angles (i.e. the $45^{\circ}$ angle information). Participants may use this $45^{\circ}$ orientation or the $45^{\circ}$ angle information to help performing the length comparison task. In fact, participants are pretty accurate in estimating vertical and horizontal orientations, and the estimated $45^{\circ}$ orientation is only slightly exaggerated (Dick and Hochstein, 1989; Durgin and Li, 2011).

The reason that we also suspect the connectedness of the two lines forming the L-shape might contribute to the HVIunderestimation is because of a recent observation made by $\mathrm{Li}$ and Durgin (2017). They asked participants to match the length of a horizontal rod to that of a vertical rod in a virtual outdoor environment. The rods were around 1-meter long. The observed HVI was only about $7 \%$ when the two rods were connected to each other forming an L-shape. Much larger illusion (about 17\%) was observed when the two rods were presented separately at different depth. If connectedness was responsible for the HVI-strength-change in Li and Durgin's experiment, it may also underlie the HVI-underestimation described above.

In the present study, three experiments were conducted to examine whether one (or both) of the aforementioned possibilities may contribute to the HVI-underestimation observed with L-shaped configurations. Experiment 1 replicated the HVI-underestimation, using similar procedure and stimuli parameters in both the length perception and length comparison tasks. This replication ruled out the possibility that the HVIunderestimation was a methodological artifact, because previous studies on HVI often used a single type of task (either the length perception or the length comparison) and the stimuli parameters were often different across studies. Experiment 2 and 3 further investigated the effect of connectedness and of the $45^{\circ}$ absolute orientation (or the $45^{\circ}$ angle) information on the observed HVI strength.

\section{Experiment 1}

Experiment 1 served as a replication of the HVIunderestimation with L-shaped configurations. Four tasks were employed including the length perception task and the traditional length comparison task, with connected L-shaped configuration. A strength difference in the HVI was expected between the two tasks. In order to make sure the HVI strength difference is not due to different task type (i.e. length perception vs. length comparison), two additional length comparison tasks with disconnected L-shaped configurations were also tested.

\section{Methods}

\section{Participants}

Thirty students (17 female) from Zhejiang University participated in this experiment for payment. All the participants had normal or corrected to normal eye sight. The experimental procedures reported in the present study were approved by the local research ethics committee and were conducted in accordance with the Declaration of Helsinki.

\section{Task and stimuli}

Four HVI tasks were tested. In the Length perception task (Fig. 1, task 1), participants estimated the length of a line (either horizontal or vertical) and compared it to a labeled size 
shown at the top-left corner of the screen. In the HVI $L$ task (Fig. 1, task 2a), participants compared the length of a horizontal line to that of a vertical line. The two lines formed a connected L-shape. In the HVI_spatial_gap task (Fig. 1, task $2 \mathrm{~b}$ ), participants compared the size of a horizontal line and that of a vertical line. A spatial gap with a random size (i.e. 1.2 $5.1 \mathrm{~cm}$ wide vertically, and $1.2 \sim 5.1 \mathrm{~cm}$ wide horizontally) separated the two lines. The HVI_temporal_gap task (Fig. 1, task 2c) was pretty similar to the HVI_spatial_gap task, with the only exception that the two lines were presented separately

Task 1: Length perception
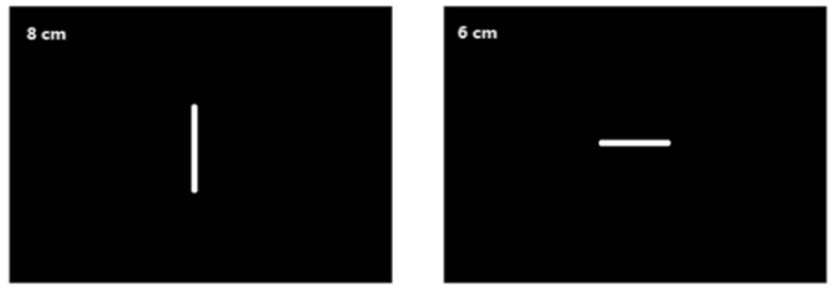

Task 2a: HVI_L
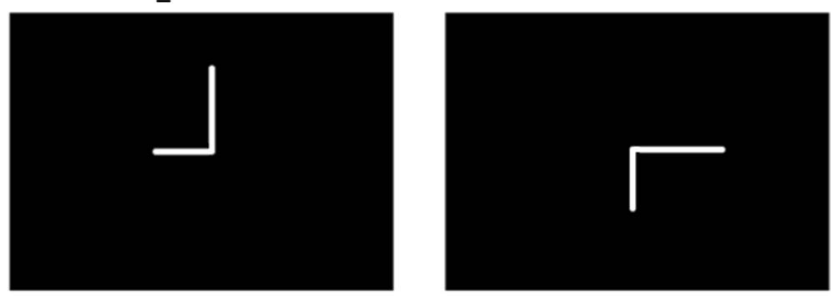

Task 2b: HVI_spatial_gap
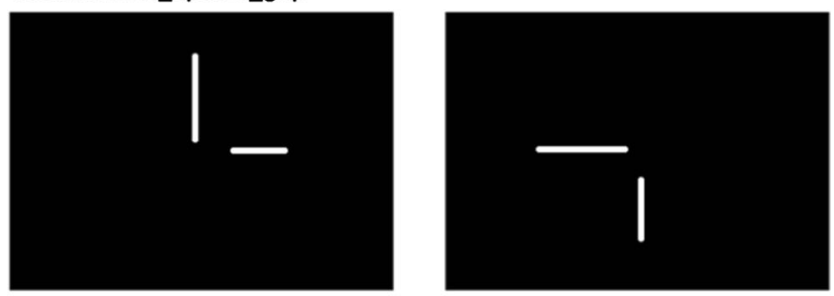

Task 2c: HVI_temporal_gap

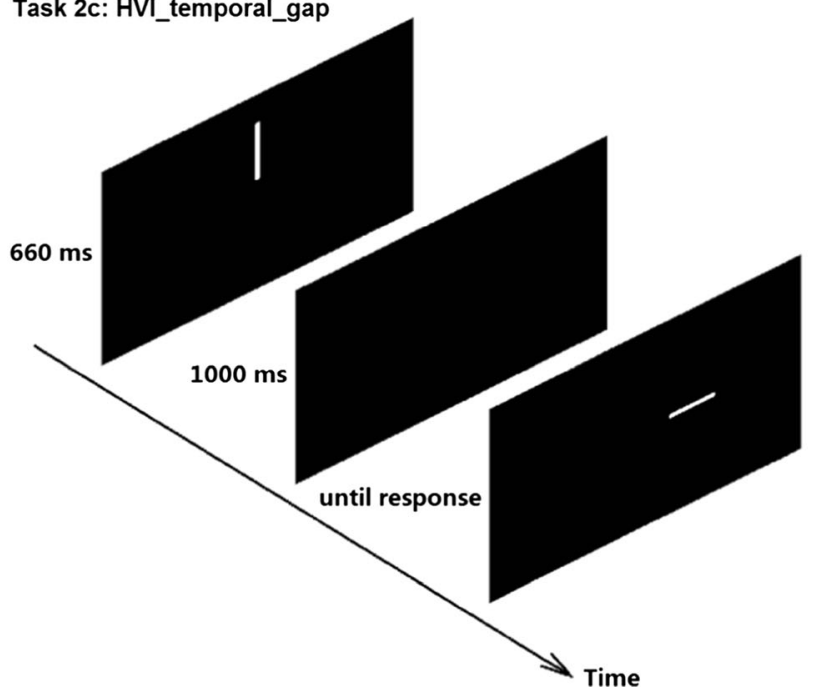

Fig. 1 Diagrams depicting the 4 tasks tested in Experiment 1. Two sample stimuli are shown for Task $1,2 \mathrm{a}$ and $2 \mathrm{~b}$ in time other than in space, with a 1 sec temporal interval. In all the length comparison tasks, the vertical line was equal likely to be on the upper or lower side of the screen, while the horizontal line was equal likely to be on the left or right side of the screen. The thirty participants were randomly assigned into three groups (ten in each). The tasks were blocked. Each group did the length perception task first, followed by one of the length comparison task. The stimuli were presented on a 21-inch CRT monitor, with a frame rate of $100 \mathrm{~Hz}$. The background was black. The width of the lines was fixed to $0.31 \mathrm{~cm}$. In the length perception task, three labeled sizes (" $4 \mathrm{~cm}$ ", "6 cm" and " $8 \mathrm{~cm}$ ") were tested. The length of the line was varied from trial to trial according to a staircase procedure (see the Procedure section). In all length comparison tasks, two orthogonal lines (a reference line and a test line) were shown in each trial. The reference line was horizontal or vertical, with a fixed size $(4,6,8 \mathrm{~cm})$. The size of the test line was adjusted from trail to trial according to the same staircase procedure. The stimuli were viewed at a distance of $70 \mathrm{~cm}$.

\section{Procedure}

In all four tasks, a logarithmic up-down staircase procedure was used to simultaneously measure the point of subjective equalities (PSEs). In the length perception task, participants judged whether the line was longer or shorter than that indicated by the labeled size. In the three length comparison tasks, participants judged which of the two lines was longer. Responses were collected via key press. The participants were allowed to take as much time as needed to make decision.

In the length perception task, six stimuli combinations were examined, i.e. 3 labeled sizes $(4,6,8 \mathrm{~cm}) \times 2$ line orientations (horizontal or vertical). Two staircases were used for each stimuli combination, with one staircase started with a length that was twice the labeled size (i.e. $8,12,16 \mathrm{~cm}$ ), and the other started with a size that was half the labeled size (i.e. 2, 3, 4 $\mathrm{cm})$. A total of twelve interleaved staircases were used. On each trial, a two-alternative forced-choice response was collected. The size of the line for the next trial in that staircase was adjusted up or down by a variable multiplicative step size, depending on the response given and the number of "turns" in that staircase so far. A "turn" is defined when two consecutive responses to the same staircase series differ. The initial step size was $3.2 \mathrm{~cm}$, which declined to $1.6 \mathrm{~cm}$ after the first turn, to $0.8 \mathrm{~cm}$ after the second turn, and to $0.4 \mathrm{~cm}$ after the third turn, where it remained thereafter. Ten "turns" for each of the twelve staircases were required to finish the test. The twelve staircases were randomly interleaved with the relative probability of a staircase being selected on any given trial being proportional to the square of the number of "turns" remaining in that staircase. This rule served to roughly synchronize the progress across all the twelve staircases. In the three length 
comparison tasks, two orthogonal lines (a reference line and a test line) were presented in each trial. The length of the reference line was fixed to 4,6 or $8 \mathrm{~cm}$. The size of the test line was adjusted up or down using the same staircase procedure. In the HVI_temporal_gap task, the vertical line and the horizontal line were equal likely to be shown first.

\section{Results}

Figure 2 shows the result of the length perception task. Mean PSE of the matched size is plotted as a function of the labeled size and line orientation. The mean PSE of matched size was systematically greater than the labeled size, indicating underestimation in the perceived size. The underestimation was stronger for the horizontal lines, e.g. a vertical line of $10.2 \mathrm{~cm}$ was matched to the " $8 \mathrm{~cm}$ " size, while a horizontal line of $11.4 \mathrm{~cm}$ was also matched to " $8 \mathrm{~cm}$ ". This anisotropy in perceived size is consistent with the horizontal vertical illusion. A 3 (labeled size: 4, 6, $8 \mathrm{~cm}$ ) x 2 (line orientation: horizontal, vertical) repeated-measures ANOVA was conducted on the PSE data of the length perception task. A statistically significant main effect was found for the labeled size, $F(2,58)$ $=168.5, p<.001, \eta^{2}=0.62$, and the line orientation, $F(1,29)$ $=62.8, p<.001, \eta^{2}=0.04$; but a statistically significant interaction was also revealed between labeled size and line orientation, $F(2,58)=12.1, p<.001, \eta^{2}=0.005$. A post hoc analysis of this interaction examined the simple main effect of the line orientation on PSE across the three labeled sizes separately, which revealed that the mean PSE for the horizontal line was significantly greater than that for the vertical line at the " $4 \mathrm{~cm}$ " size, $F(1,29)=25.6, p<.001, \eta^{2}=0.03$, the " 6 cm" size, $F(1,29)=30.8, p<.001, \eta^{2}=0.05$, and also the "8 $\mathrm{cm}$ " size, $F(1,29)=65.3, p<.001, \eta^{2}=0.05$. This interaction is arising from the fact that the difference in length estimation between vertical and horizontal lines is greater in the " $8 \mathrm{~cm}$ " than " $6 \mathrm{~cm}$ " than " $4 \mathrm{~cm}$ " cases.

Because each participant first did the length perception task, HVI was estimated from the length perception data for

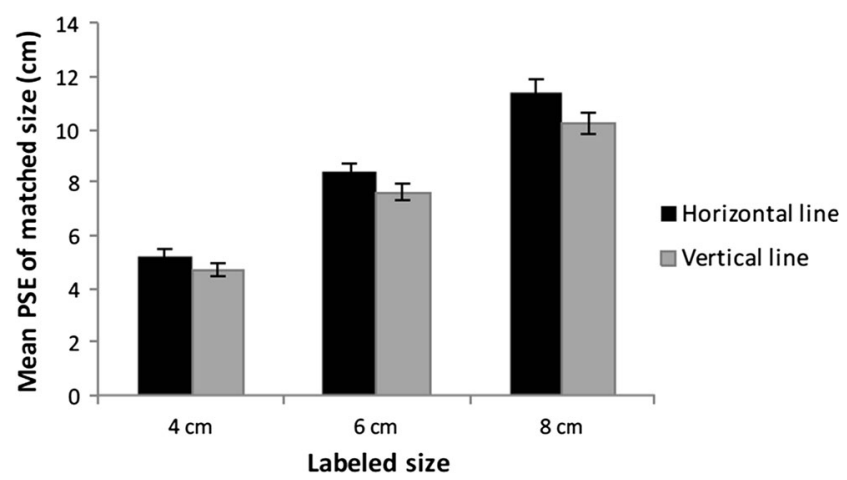

Fig. 2 Results of the length perception task in Experiment 1. The mean PSE of matched line size is plotted as a function of the labeled size and line orientation. Standard errors are shown

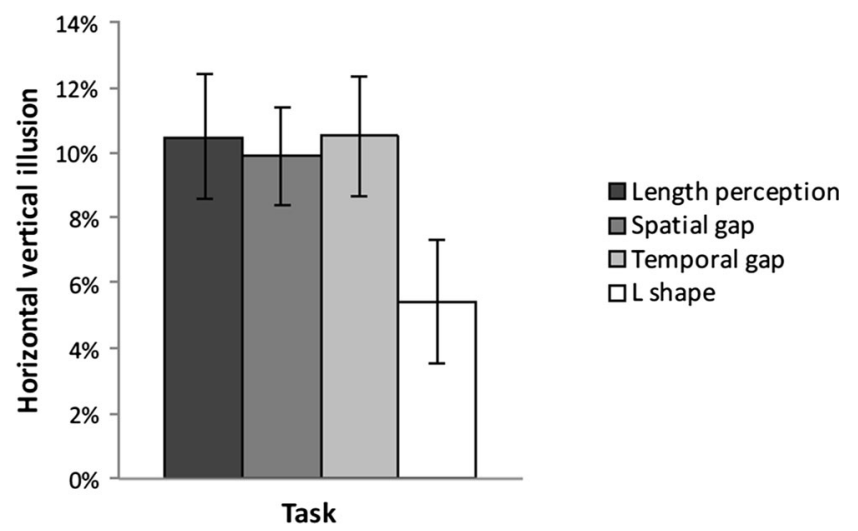

Fig. 3 Mean magnitude of the horizontal vertical illusion observed in the four tasks examined in Experiment 1. The error bars represent the 95\% confidence intervals

each participant, by making the PSE for the vertical line the standard and computing the percent difference based on the PSE of the horizontal line. A one-way repeated-measures ANOVA with labeled size as the only factor was conducted on the estimated HVI data. No statistically significant main effect of labeled size was found, $F(2,58)=0.039, p=.921, \eta^{2}$ $=0.001$. That is, the HVI observed in the length perception task did not vary in magnitude with the labeled-sizes tested. This result showed that the observed interaction between labeled size and line orientation went away when data were normalized to percent difference, which suggested a Weberesque scaling effect. The HVI data were then collapsed across labeled size. The mean HVI was 10.5\% (Fig. 3, left-most bar).

The HVI observed in the length perception task provided a baseline $(10.5 \%)$ for the magnitude of HVI in our participants. We then examined the HVIs observed in the length comparison tasks. The HVI of each participant at each reference line size $(4,6,8 \mathrm{~cm})$ was obtained for each length comparison task, by comparing the PSE for the test line size to the corresponding reference line size. A mixed-effects ANOVA with the reference line size $(4,6,8 \mathrm{~cm})$ as within-subject factor and with the task type (HVI_spatial_gap, HVI_temporal_gap, HVI_L) as between-subject factor was conducted on the HVI data from the length comparison tasks. A statistically significant main effect of task was found, $F(2,27)=4.59, p=.019, \eta^{2}=$ 0.201 . No significant main effect of reference line size was found, $F(2,54)=1.78, p=.179, \eta^{2}=0.017$, nor significant interaction between task and reference line size was seen, $F(4$, $54)=1.29, p=.287, \eta^{2}=0.025$. A pairwise t-test with Bonferroni correction revealed that the mean HVI observed in the HVI_L task $(\mathrm{M}=5.4 \%)$ was significantly smaller than that observed in the HVI_spatial_gap task $(\mathrm{M}=9.9 \%)(p$ $<.005)$ and than that observed in the HVI temporal gap task $(\mathrm{M}=10.5 \%)(p<.001)$, but the difference in the mean HVI observed between the HVI_spatial_gap task and HVI_temporal_gap task was not significant $(p>.50)$. Three one-sample t-tests were conducted to compare the HVI 
observed in the length comparison tasks to the HVI baseline $(10.5 \%)$ observed in the length perception task. The mean HVI in the HVI_L task was significantly smaller than $10.5 \%, t(9)=-3.33, p<.01$, Cohen's $d=1.05$; but no significant difference was seen between $10.5 \%$ and the mean HVI from the HVI_spatial_gap task, $t(9)=-0.51, p=.621$, Cohen's $d$ $=0.16$; nor between $10.5 \%$ and the mean HVI from the HVI_temporal_gap task, $t(9)=-0.018, p=.986$, Cohen's $d$ $=0.006$.

\section{Discussion}

In Experiment 1, we compared the horizontal vertical illusion reflected from a length perception task to that reflected from a length comparison task with three different stimuli configurations (i.e. the two lines were connected and arranged in an Lshape, or the two lines were separated by a spatial gap, or the two lines were separated by a temporal gap). If the HVI reflects a perceptual anisotropy between horizontal and vertical sizes, the illusion seen in the length perception task actually provides a baseline for the magnitude of the illusion. The present results showed that the mean illusion was $10.5 \%$ for our participants. The illusion observed in the length comparison tasks varied with stimuli configuration. The strength of the illusion was no different from the baseline when the two lines were separated either in space or in time. In contrast, the illusion was significantly reduced when the stimuli were configured in a connected L-shape. These results suggested that the horizontal vertical illusion is underestimated in connected L-shaped configurations.

As mentioned in the introduction, we suspect two possible factors that may contribute to the HVI-underestimation in Lshaped configurations: The $45^{\circ}$ absolute orientation $/ 45^{\circ}$ angle information contained in the isosceles upright L-shape (when the two lines match in size), and the connectedness of the two lines forming the L-shape. Experiment 2 examined the possible effect of the $45^{\circ}$ absolute orientation information, while Experiment 3 examined the effect of the $45^{\circ}$ angle information. The connectedness was manipulated in both Experiment 2 and 3 .

\section{Experiment 2}

The purpose of Experiment 2 was to examine whether the HVI-underestimation observed in the connected L-shaped configuration in Experiment 1 was due to the $45^{\circ}$ absolute orientation information contained in the upright L-shape or was caused by the connectedness between the two lines. When the length of the two lines matches, the implicit line connecting the two ends of the upright L-shape would become $45^{\circ}$ tilted from horizontal. Studies on orientation perception suggested that people are pretty accurate at estimating
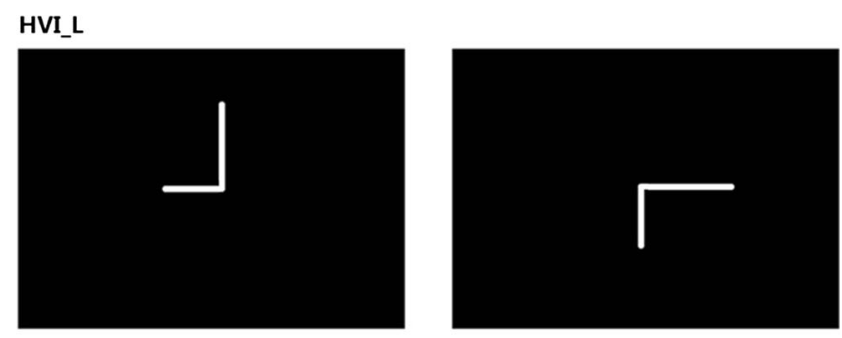

HVI_spatial_gap
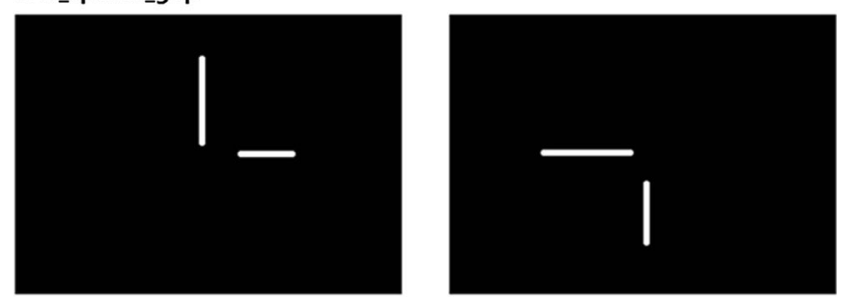

HVI L $15^{\circ}$
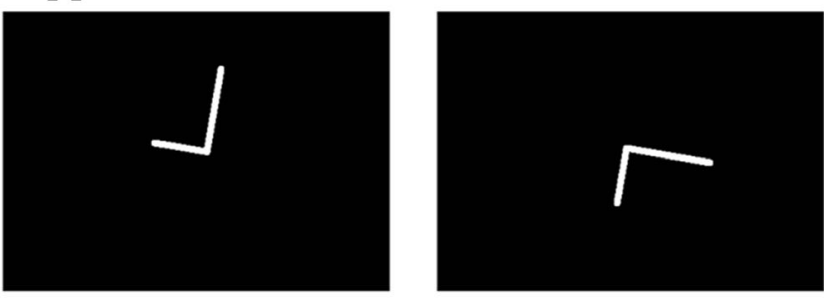

HVI_spatial_gap_15 ${ }^{\circ}$
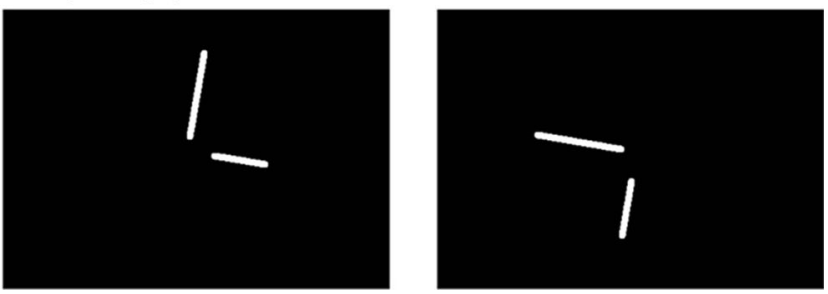

Fig. 4 Diagrams depicting the four tasks used in Experiment 2. Two sample stimuli are shown for each task

horizontal and vertical orientations, and the estimated $45^{\circ}$ orientation is also only slightly exaggerated (Dick and Hochstein, 1989; Durgin and Li, 2011). Therefore, participants may be able to use this orientation information to help them better performing the length comparison task. In Experiment 2, this absolute orientation information was made helpless for the length comparison task, by rotating both lines $15^{\circ}$ clockwise.

\section{Methods}

\section{Participants}

Twelve students from Zhejiang University participated in Experiment 2. All the participants had normal or corrected to normal eye sight. None of them participated in Experiment 1. 


\section{Task, stimuli, and procedure}

A within-subject design with 2 orientations (upright or $15^{\circ}$-tilt) $\mathrm{x} 2$ connectivity types (connected or spatially-separated) was used, generating four tasks (Fig. 4). The HVI_L and the HVI_spatial_gap tasks were identical to those tested in Experiment 1 . The HVI_L_15 $5^{\circ}$ and the HVI_spatial_gap_15 tasks were new. The new tasks were modified from the old ones. The only difference between the new tasks and the corresponding old tasks was that the whole stimuli were rotated $15^{\circ}$ clockwise in the new ones. All the other aspects of the new tasks, e.g. stimuli feature, task procedure, etc., were identical to those used in the old tasks. The 4 tasks were blocked so that the participants did one task at a time. The two old tasks with the upright stimuli and the two new tasks with the $15^{\circ}$ tilted stimuli were further grouped, so that the first two tasks did by each participant were always either the two old tasks or the two new tasks. The order of the two groups (new vs. old tasks) was counterbalanced across participants. The order of the two tasks within each group was also counterbalanced across participants.

\section{Results}

The illusion of perceived ratio between the two lines for each reference line size $(4,6,8 \mathrm{~cm})$ was obtained for each participant in each of the four tasks by comparing the PSE for the test line size and the corresponding reference line size. A 3 (reference line size: $4,6,8 \mathrm{~cm}$ ) x 2 (orientation: upright, $15^{\circ}$ tilted) $\mathrm{x}$ 2 (connectivity type: connected, spatially separated) repeatedmeasures ANOVA was conducted on the illusion data. The ANOVA revealed a statistically significant main effect of orientation, $F(1,11)=7.83, p=.017, \eta^{2}=0.042$, and a statistically significant main effect of connectivity type, $F(1,11)=$ 97.2, $p<.0001, \eta^{2}=0.466$, but it did not find a statistically significant main effect of reference line size, $F(2,22)=2.21, p$ $=.133, \eta^{2}=0.024$. The ANOVA also found a statistically significant interaction between orientation and connectivity type, $F(1,11)=6.04, p=.032, \eta^{2}=0.030$, but no other 2 way or 3-way interaction was found significant. The illusion data were collapsed across the reference line size. The mean illusion for the four tasks are shown in Fig. 5. A post hoc analysis revealed that the mean illusion is significantly reduced in the connected configuration relative to that in the spatially-separated configuration both for the upright orientation, $F(1,11)=124.6, p<.001, \eta^{2}=0.684$, and for the $15^{\circ}$-tilt orientation, $F(1,11)=31.9, p<.001, \eta^{2}=0.479$. In contrast, the mean illusion was significantly smaller in the $15^{\circ}$-tilt orientation only when the two lines were separated, $F(1,11)$ $=16.3, p<.01, \eta^{2}=0.225$, but not when they were connected, $F(1,11)=0.12, p=.735, \eta^{2}=0.002$. These results replicated the basic finding in Experiment 1 and suggested that even when the $45^{\circ}$ absolute orientation information was made useless, the illusion strength was still significantly reduced when the two lines were connected to each other.

\section{Discussion}

In Experiment 2, we studied whether the $45^{\circ}$ absolute orientation information embedded in the upright connected L-shaped configuration when the two lines match in size could be the cause of the L-shape induced HVI-underestimation seen in Experiment 1. This orientation information was intentionally made useless for length comparison by rotating both lines $15^{\circ}$ clockwise. If the orientation information were the main cause of the HVI-underestimation, we may expect a relatively large illusion (about $10 \%$ ) in the HVI_L_1 $15^{\circ}$ task because the $45^{\circ}$ orientation information was not useful in this task. However, the result showed that the illusion in the HVI_L_ $15^{\circ}$ task was less than $5 \%$, which suggested the implicit $45^{\circ}$ orientation information was not the cause of the HVI-underestimation. In contrast, the connectedness manipulation strongly affected the illusion, which suggested that it might be the real cause of the HVIunderestimation observed in Experiment 1.

\section{Experiment 3}

Experiment 2 excluded the possibility that the L-shape induced HVI-underestimation was caused by the use of the $45^{\circ}$ absolute orientation information. However, in Experiment 2, the two lines was still perpendicular to each other so that the connected L-shape formed an implicit isosceles right-triangle when the two lines matched in size. It is still possible that the $45^{\circ}$ inner angles contained in the isosceles right-triangle could be helpful for the length comparison task. To examine this possibility, in Experiment 3, only the vertical line was rotated $15^{\circ}$ clockwise while the horizontal line remained horizontal, so that the two lines formed an angle of either $75^{\circ}$ or $105^{\circ}$.

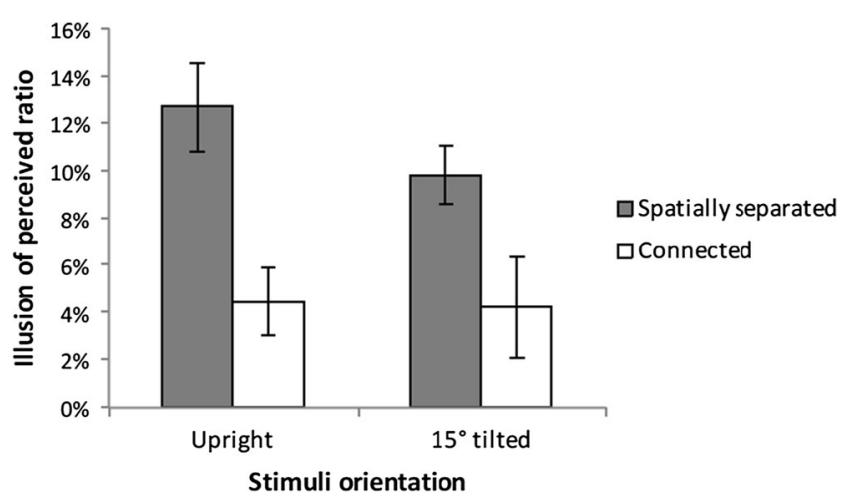

Fig. 5 Mean illusion of the perceived ratios observed in the four tasks tested in Experiment 2. The error bars represent the 95\% confidence intervals 


\section{Methods}

\section{Participants}

Twelve students from Zhejiang University participated in Experiment 3. All the participants had normal or corrected to normal eye sight. None of them participated in Experiment 1 or 2.

\section{Task, stimuli, and procedure}

Three tasks used in Experiment 1 (length perception, HVI_L, and HVI_spatial_gap) were adopted with modification in Experiment 3. They were called Length perception, Length comparison connected, and Length comparison separated respectively (Fig. 6). The major difference between the tasks from Experiment 1 and the new tasks was that, in the new tasks, the vertical line was replaced by a line tilted $15^{\circ}$ clockwise from vertical. Moreover, in the length comparison tasks of Experiment $1 \& 2$, the position between the vertical line and the horizontal line was not fixed. That is, the vertical line was presented above or below the horizontal line while the horizontal line could be presented to the left or right to the vertical line, generating 4 possible combinations that were randomly mixed in each block. In contrast, in the length comparison tasks in Experiment 3, the tilted line was always above the

\section{Length perception}
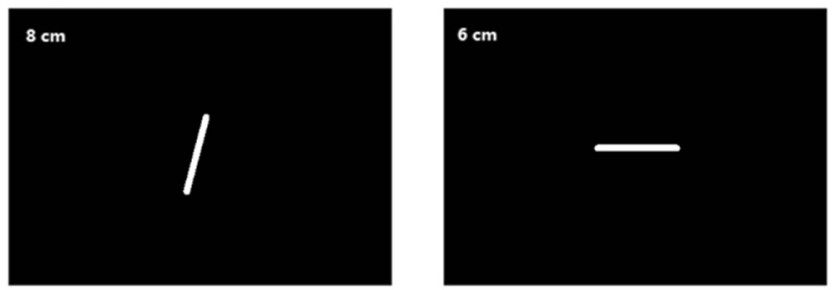

Length comparison connected
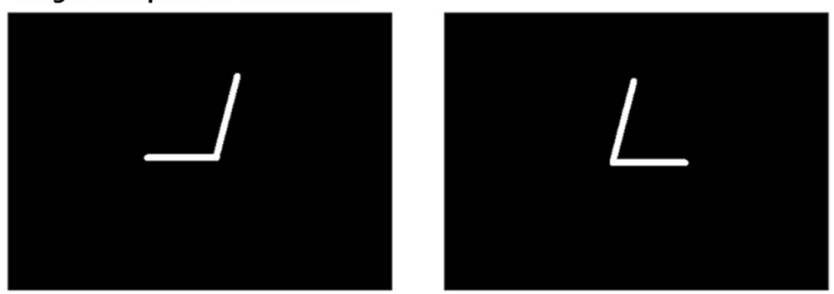

Length comparison separated
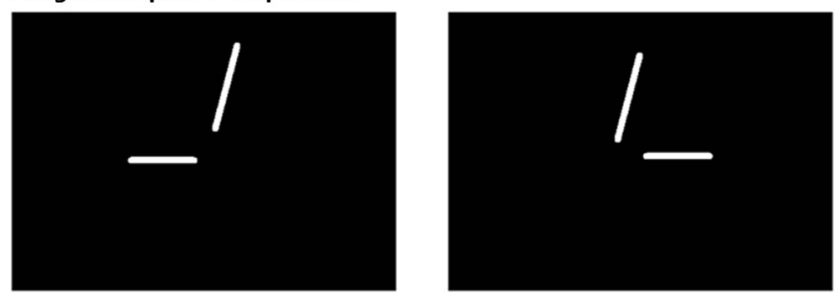

Fig. 6 Diagrams depicting the tasks used in Experiment 3. All possible line position combinations are shown for the length comparison tasks horizontal line so that only two possible relative line positions were tested, i.e. the two lines formed an angle of either $75^{\circ}$ or $105^{\circ}$. Thus, the two length comparison tasks contained altogether four possible stimuli configurations (Fig. 6, middle and bottom panels). The stimuli configuration was blocked. The reason for this blocked design was because previous study showed that the HVI strength was greater in obtuse-angle Lshape than that in acute-angle L-shape (Cormack and Cormack, 1974). A within-subject design was used. Each participant did all three tasks. The length perception task was always conducted in the first block. The two length comparison tasks were conducted consecutively. Each task contained two blocks, with one stimuli configuration $\left(75^{\circ}\right.$ or $\left.105^{\circ}\right)$ being tested in a block. The order of the two comparison tasks was counterbalanced across participants. For each task, the order of the two stimuli configuration was also counterbalanced. The procedures in Experiment 3 were identical to those used in Experiment 1.

\section{Results}

As that in Experiment 1, the Length perception task in Experiment 3 also provided a baseline for the strength of the illusion in perceived ratios. The magnitude of the illusion for each participant at each labeled size was obtained by making the PSE for the tilted line the standard and computing the percent difference based on the PSE of the horizontal line. A repeated-measures ANOVA with labeled size as the only factor was conducted on the illusion data. No statistically significant main effect of labeled size was found, $F(2,22)=0.267, p$ $=.768, \eta^{2}=0.008$. The data were then collapsed across labeled size. The mean illusion for all participants was $10.0 \%(95 \%$ confidence intervals: $5.26 \%$ to $14.76 \%$ ), quite similar to the HVI baseline (10.5\%) seen in Experiment 1.

The illusions of perceived ratio between the tilted line and horizontal line for each reference line size $(4,6$, or $8 \mathrm{~cm})$ with all connectivity type (connected, spatially separated) x stimulus shape (acute-angle, obtuse-angle) combinations were obtained for each participant, by comparing the PSE of the test line size to the corresponding reference line size. A 3 (reference line size: 4,6 , or $8 \mathrm{~cm}$ ) x 2 (connectivity type: connected, spatially separated) x 2 (stimulus shape: acute-angle, obtuseangle) repeated-measures ANOVA was conducted on the illusion data. The ANOVA found a statistically significant main effect of reference line size, $F(2,22)=10.5, p<.001, \eta^{2}=$ 0.074 , a statistically significant main effect of connectivity type, $F(1,11)=18.82, p<.005, \eta^{2}=0.155$, and a marginally significant main effect of stimulus shape, $F(1,11)=4.64, p$ $=.054, \eta^{2}=0.047$. The ANOVA also revealed statistically significant interactions between reference line size and connectivity type, $F(2,22)=9,93, p<.001, \eta^{2}=0.018$, and between reference line size and stimulus shape, $F(2,22)=4.58$, $p=.022, \eta^{2}=0.008$. No other 2 -way or 3-way interaction was 

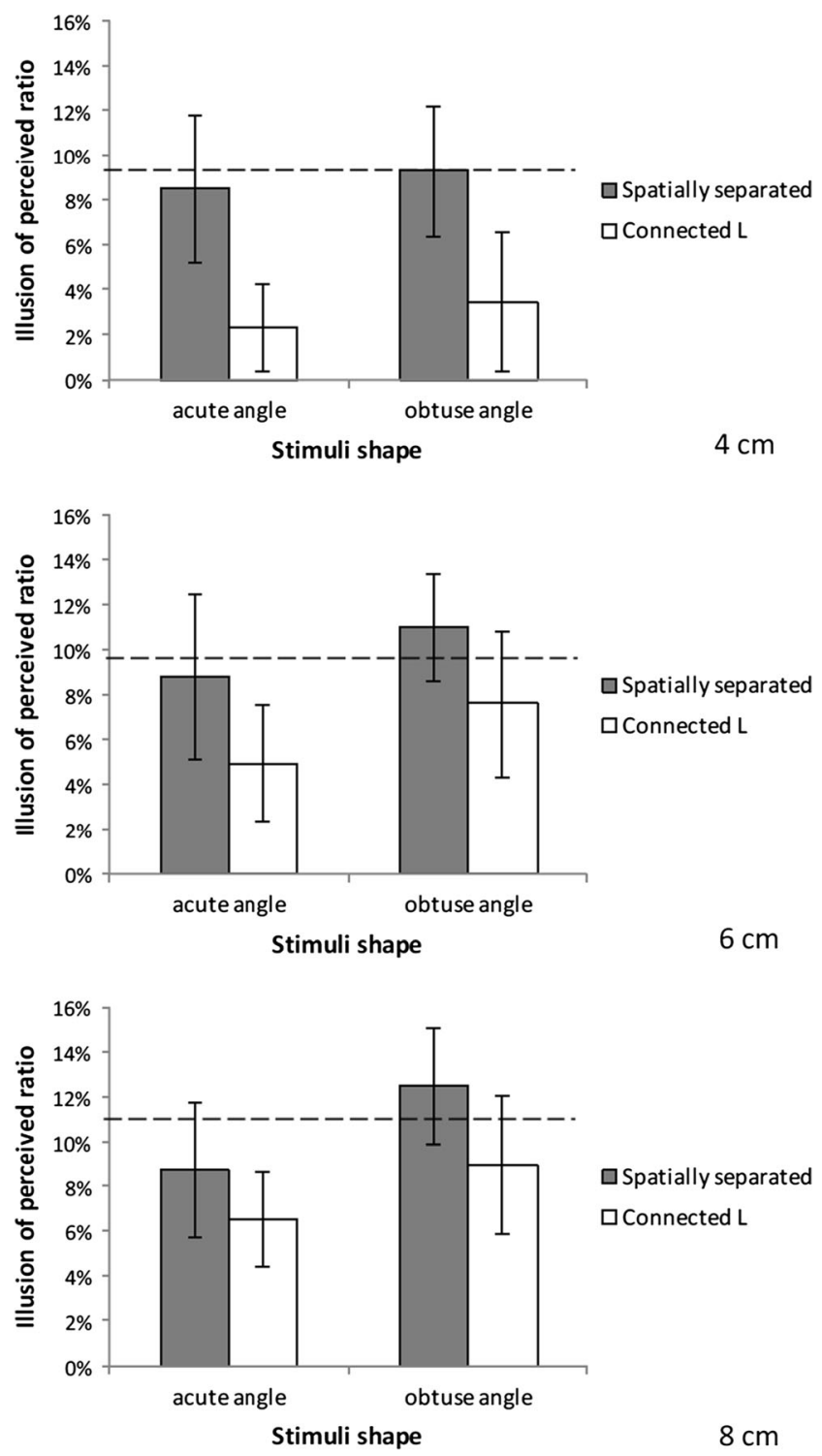

Fig. 7 Mean illusion of the perceived ratio between the tilted line and the horizontal line found in the two length comparison tasks in Experiment 3. The dashed lines represent the (baseline) illusion level seen in the length perception task. The error bars represent the $95 \%$ confidence intervals

significant. The data were then analyzed across reference line size separately. Three two-way repeated-measures ANOVAs with the connectivity type and the stimulus shape as factors were conducted on the illusion data for the three reference line sizes respectively. For the $4 \mathrm{~cm}$ reference line size, a statistically significant main effect of connectivity type was found, $F(1,11)=54.7, p<.001, \eta^{2}=0.28$; no significant main effect of stimulus shape was seen, $F(1,11)=0.96, p=.349, \eta^{2}=$ 0.01; nor any significant interaction between the connectivity type and stimulus shape was found, $F(1,11)=0.03, p=.87, \eta^{2}$ $<0.001$. For the $6 \mathrm{~cm}$ reference line size, statistically significant main effects were found for the connectivity type, $F(1,11)$ $=10.9, p<.01, \eta^{2}=0.11$, and for the stimulus shape, $F(1,11)$ $=5.6, p=.037, \eta^{2}=0.05$; but no significant interaction was seen between connectivity type and stimulus shape, $F(1,11)=$ $0.02, p=.882, \eta^{2}<0.001$. For the $8 \mathrm{~cm}$ reference line size, statistically significant main effects were found for the connectivity type, $F(1,11)=5.69, p=.036, \eta^{2}=0.09$, and stimulus shape, $F(1,11)=6.4, p=.028, \eta^{2}=0.1$; but no significant interaction between connectivity type and stimulus shape was found, $F(1,11)=0.46, p=.51, \eta^{2}=0.005$. Mean illusion of the perceived ratio is plotted as a function of the connectivity type and the stimulus shape for each reference line size (Fig. 7). For comparison, the illusion levels (baselines) from the length perception task are also shown in Fig. 7 as dashed lines.

\section{Discussion}

In Experiment 1, we found that the horizontal vertical illusion was significantly underestimated when a connected L-shaped configuration was used in the length comparison task. When the two lines match in size, the L-shaped configuration forms an implicit isosceles right-triangle that contains two $45^{\circ}$ inner angles. We suspected that participants might use this angle information to help better performing the length comparison task. In Experiment 3, we tested this possibility by rotating only the vertical line $15^{\circ}$ clockwise. If the L-shape induced HVI-underestimation in Experiment 1 was indeed due to the use of this $45^{\circ}$ angle information, we should expect comparable illusion strength in all the tested conditions in Experiment 3 , because the $45^{\circ}$ angle information was no longer helpful for the length comparison task. The results showed that the strength of the illusion was strongly affected by the connectedness, which suggested that it was probably the connectedness between the two lines that caused the observed L-shape induced HVI-underestimation.

\section{General discussion}

Since Fick first reported the horizontal vertical illusion in 1851, this illusion has been extensively studied by many researchers. The most common way of demonstrating the HVI is perhaps to use the length comparison paradigm, with various line configurations, such as L-shape, inverted T-shape, cross, dissected T-shape, etc. (Avery and Day 1969; Cormack and Cormack 1974; Houck et al 1972; Künnapas 1955, 1957; Landwehr 2015; Prinzmetal and Gettleman 1993). In addition to the length comparison paradigm, the HVI was also demonstrated by length perception per se, i.e. the perceived length of a 2D line varies with the orientation of the line (e.g. Armstrong and Marks 1997; Pollock and Chapanis 1952; Teghtsoonian 1972; Verrillo and Irvin 1979). The results from previous studies suggested that both the line configuration and the task paradigm can affect the strength of HVI. For example, the HVI observed in the length perception task was often larger than $10 \%$, while the HVI found in the length comparison tasks was only about $5 \%$ for 
the L-shaped configurations and was about $10 \%$ for the inverted-T shaped configurations. Whereas many studies examined why HVI found in the L-shape was different from that seen in the inverted-T shape (e.g. Avery and Day 1969; Charras and Lupiáñez 2010), to our knowledge, no single study has directly investigated whether and why the HVI in the L-shaped length comparison task is smaller than that found with the length perception technique.

In the present study, we examined whether and why the HVI is underestimated in the L-shaped configurations. In Experiment 1, we compared the HVI from the length perception task to those from the three length comparison tasks (i.e. the two lines were arranged in a connected L-shape, or the two lines were separated by a spatial gap or by a temporal gap). The results showed that the HVI strength observed in the length perception task (about 10\%) was no different from that in the length comparison task when the two lines were separated. In contrast, when the two lines were arranged in a connected L-shape, the HVI strength reduced to about 5\%. These findings suggested that it was the connected L-shape configuration per se rather than the task paradigm (i.e. length perception vs. length comparison) that caused the HVI-underestimation. We then investigated whether the HVIunderestimation was caused by the connectedness between the two lines or was due to an implicit use of the $45^{\circ}$ absolute orientation information (Experiment 2) or the $45^{\circ}$ angle information (Experiment 3 ) embedded in the upright isosceles Lshape. The results showed that even when this $45^{\circ}$ absolute orientation and the $45^{\circ}$ angle information was made useless for the length comparison task, the illusion was still significantly reduced when the lines were connected relative to when they were separated. The results from all the three experiments provided converging evidence suggesting that the primary contribution to the HVI-underestimation observed in the Lshaped configuration was probably the connectedness of the two lines forming the L-shape.

If the connectedness between the two lines forming the Lshape substantially reduces the HVI from $10 \%$ to $5 \%$, then why the HVI is still $10 \%$ in the inverted-T shape, in which the two lines are also connected to each other? There are two possibilities. First, as some studies suggested, there might be two illusions in the inverted-T shape, i.e. a horizontal vertical illusion and a bisection illusion (Charras and Lupiáñez 2010). It is possible that the effect of the bisection illusion just cancelled out the HVI-underestimation caused by the connectedness in the inverted-T shape.

There is another way to explain why the HVI observed in the inverted-T shape is still $10 \%$. There is evidence suggesting that the L-shape is perceived as a single object, whereas the inverted-T shape is perceived as two objects. For example, Feldman (2007) studied the perceptual grouping of line segment pairs arranged in 12 different configurations, including the L-shape and T-junction. Based on the idea of "object benefit", Feldman measured the response time for fast comparison of the size of two dots that superimposed on the line segments. The response time was longer when the dots were superimposed on the line segments of a T-junction as compared to when they were superimposed on the segments of an L-shaped configuration, which suggested the two segments of the L-shape was perceived as a single object but the two segments of the T-junction was perceived as separate objects. The same conclusion can also be predicted by a formal quantitative model (see van der Helm 2011; van Lier, van der Helm and Leeuwenberg 1994). If the two lines in the inverted-T shape are perceived as separate objects, the corresponding HVI strength should be similar to that seen in the HVI_spatial_gap task in Experiment 1. Indeed, the HVI observed in the HVI_spatial_gap task was about $10 \%$, which was consistent with the common finding for the HVI in the inverted-T shape configurations. This explanation also agrees with the claim that within-object comparisons are easier (faster, more accurate) than between-objects comparisons (Behrmann, Zemel, and Mozer, 1998). Moreover, a recent HVI study used dissected T-shape showed that for upright dissected T-shape, the observed HVI strength was still around 10\% (Landwehr 2015, pp. 2150).

Whether the HVI-underestimation in the L-shaped configuration, as compared to the HVI from the inverted-T shape configuration, was due to the difference between the withinobject comparison and between-object comparison may require future investigation. The present study emphasizes that the HVI is substantially underestimated when a connected Lshaped configuration is used to evaluate the illusion. Future studies on the horizontal vertical illusion must be cautious if connected L-shaped configuration is to be used as the visual stimuli.

Acknowledgements We thank Peter van der Helm and another anonymous reviewer for their helpful comments on an early version of this paper. We thank Tenfei Wang for his help on the statistical analysis of the data. This study was supported by the National Natural Science Foundation of China (31671129) and by the Fundamental Research Funds for the Central Universities (2016QNA3017), and by the Zhejiang Provincial Natural Science Foundation of China (LY15C090002).

\section{References}

Armstrong, L., \& Marks, L. E. (1997). Differential effects of stimulus context on perceived length: Implications for the horizontal-vertical illusion. Perception \& Psychophysics, 59(8), 1200-1213.

Avery, G. C., \& Day, R. H. (1969). Basis of the horizontal-vertical illusion. Journal of Experimental Psychology, 81(2), 376-380.

Behrmann, M., Zemel, R. S., \& Mozer, M. C. (1998). Object-based attention and occlusion: Evidence from normal participants and a computational model. Journal of Experimental Psychology: Human Perception and Performance, 24(4), 1011. 
Charras, P., \& Lupiáñez, J. (2010). Length perception of horizontal and vertical bisected lines. Psychological Research PRPF, 74(2), 196206.

Cormack, E. O., \& Cormack, R. H. (1974). Stimulus configuration and line orientation in the horizontal-vertical illusion. Perception \& Psychophysics, 16(2), 208-212.

Dick, M., \& Hochstein, S. (1989). Visual orientation estimation. Perception \& Psychophysics, 46, 227-234.

Durgin, F. H., \& Li, Z. (2011). The perception of 2D orientation is categorically biased. Journal of Vision, 11(8), 13: 1-10.

Feldman, J. (2007). Formation of visual "objects" in the early computation of spatial relations. Perception \& Psychophysics, 69(5), 816827.

Fick, A. (1851). De errone quodam optic asymmetria bulbi effecto [On asymmetric error in the effective optic sphere]. Marburg: Koch.

Finger, F. W., \& Spelt, D. K. (1947). The illustration of the horizontalvertical illusion. Journal of Experimental Psychology, 37(3), 243250 .

Girgus, J. S., \& Coren, S. (1975). Depth cues and constancy scaling in the horizontal - vertical illusion: The bisection error. The Canadian Journal of Psychology, 29, 59-65.

Gregory, R. L. (1963). Distortion of visual space as inappropriate constancy scaling. Nature, 199, 678-780.

Houck, R. L., Mefferd, R. B., \& Greenstein, G. J. (1972). Influence of a visual frame and vertical-horizontal illusion on shape and size perception. Journal of Experimental Psychology, 96(2), 273-279.

Künnapas, T. M. (1955). An analysis of the" vertical-horizontal illusion". Journal of Experimental Psychology, 49, 134-139.

Künnapas, T. M. (1957). The vertical-horizontal illusion and the visual field. The Journal of Experimental Psychology, 53, 405-407.

Landwehr, K. (2015). Titchener's $\perp$ dissected. Attention, Perception \& Psychophysics, 77(6), 2145-2152.
Pollock, W. T., \& Chapanis, A. (1952). The apparent length of a line as a function of its inclination. Quarterly Journal of Experimental Psychology, 4(4), 170-178.

Prinzmetal, W., \& Gettleman, L. (1993). Vertical-horizontal illusion: One eye is better than two. Perception \& Psychophysics, 53(1), 81-88.

Spector, R. H. (1990). Visual fields. In H. K. Walker, W. D. Hall, \& J. W. Hurst (Eds.), Clinical methods: The history, physical, and laboratory examinations. Boston: Butterworths.

Teghtsoonian, M. (1972). Apparent length as a function of tilt does not depend on orientation of the standard. Journal of Experimental Psychology, 94(2), 191-197.

van der Helm, P. A. (2011). Bayesian confusions surrounding simplicity and likelihood in perceptual organization. Acta Psychologica, 138(3), 337-346.

van Lier, R., Van Der Helm, P., \& Leeuwenberg, E. (1994). Integrating global and local aspects of visual occlusion. Perception, 23(8), 883903.

Verrillo, R. T., \& Irvin, G. (1979). Absolute estimation of line length as a function of orientation and contrast polarity. Sensory Processes, 3, 261-274.

Williams, P. A., \& Enns, J. T. (1996). Pictorial depth and framing have independent effects on the horizontal-vertical illusion. Perception, 25, 921-926.

Wolfe, U., Maloney, L. T., \& Tam, M. (2005). Distortions of perceived length in the frontoparallel plane: Tests of perspective theories. Perception and Psychophysics, 67, 967-979.

Zhi Li and Frank H. Durgin (2017), A large-scale horizontal-vertical illusion produced with small objects separated in depth, Journal of Experimental Psychology: Human Perception and Performance (in press) 